\title{
A test for the detection of vegetation on extrasolar planets: detection of vegetation in Earthshine spectrum and its diurnal variation
}

\author{
Danielle Briot $^{1}$, Karim Agabi ${ }^{2}$, Eric Aristidi ${ }^{2}$, Luc Arnold ${ }^{3}$, Patrick \\ François $^{4,1}$, Pierre Riaud ${ }^{5}$, Patrick Rocher ${ }^{1}$ and Jean Schneider ${ }^{5}$ \\ ${ }^{1}$ Observatoire de Paris-Meudon, 61 avenue de l'Observatoire, F-75014 Paris, France \\ email: Danielle.Briot@obspm.fr \\ ${ }^{2}$ Université de Nice - Sophia Antipolis, 28 avenue Valrose, F-06108 Nice cedex 2, France \\ ${ }^{3}$ Observatoire de Haute-Provence-CNRS, F-04870 Saint Michel l'Observatoire, France \\ ${ }^{4}$ European Southern Observatory (ESO), 3107 Av. de Cordova, Vitacura, Santiago 19, Chile \\ ${ }^{5}$ Observatoire de Paris-Meudon, 5 place Jules Janssen, F-92195 Meudon, France
}

\begin{abstract}
The search for life in extraterrestrial planets is to be tested first with the only planet known to shelter life. If the planet Earth is used as an example to search for a signature of life, the vegetation is one of its possible detectable signature, using the Vegetation Red Edge due to chlorophyll in the near infrared $(0.725 \mu \mathrm{m})$. We focus on the test of the detectability of vegetation in the spectrum of Earth seen as a simple dot, using the reflection of the global Earth on the lunar surface, i.e., Earthshine. On the Antartic, the Earthshine can be seen during several hours in a day (not possible at our latitudes) and so variations due to different parts of Earth, that is to say oceans and continents facing the Moon could be detected.
\end{abstract}

Keywords. Earth, Moon, astrobiology, techniques : spectroscopy, vegetation red edge, biomarker, Earthshine, exolife

\section{Introduction}

It could be hoped that in few years (e.g., beyond 2015) we will search for detection of life in terrestrial extrasolar planets. Life on extrasolar planets will probably present unusual and unknown forms. However, as we know nothing about these forms of life, we look for indices of presence of life similar to the one we know on Earth. Firstly, we explore classical biosignatures like $\mathrm{H}_{2} \mathrm{O}, \mathrm{CO}_{2}, \mathrm{O}_{3}$ and $\mathrm{O}_{2}$, but it is also interesting in visible wavelengths to search how vegetation can be distinguished on a planet seen from space.

\section{Detection of vegetation}

Vegetation spectrum presents an increase at $0.5 \mu \mathrm{m}$ in the green range, which implies that plants are seen as green, but mostly a very sharp rise at $0.725 \mu \mathrm{m}$, known as the Vegetation Red Edge (VRE, Arnold et al. 2002), the signature of photosynthetic plants. The Vegetation Red Edge can be much more easily detected than the bump at $0.5 \mu \mathrm{m}$, and this signature corresponds hardly to other elements than chlorophyll. The search for vegetation on exoplanets should be tested with the only planet known to shelter life. Vegetation can be detected on the planet Earth from a spacecraft as done by Sagan et al. (1993) using the Galileo spacecraft but in that case, vegetation has been detected vertically, and obliquity, limb effects, nor cloud cover have been considered. Earth has to be observed as a whole like we see a extrasolar planet, i.e., as a point source. Presently, no distant spacecraft has the cability to take a spectrum of the whole Earth. 


\section{Earthshine}

Another possibility is to use the Moon as a giant reflector and to observe ashen light or Earthshine. Earthshine can be seen on the dark part of the Moon during the first or the last days of the lunar cycle. This corresponds to a Earth light on the Moon. The light of the Sun arrives on Earth, is reflected by Earth, arrives on the Moon, is reflected by the Moon and comes back on Earth. The light coming from the different parts on Earth is blended and thus, as in the case of an exoplanet, seen integrated. Then: $[$ Earthshine Spectrum $]=[$ Solar Spectrum $] \times[$ Earth Albedo $] \times[$ Moon Albedo $]$ and transmitted three times through Earth atmosphere; and: [Moonligh spectrum] $=$ [Solar Spectrum $] \times[$ Moon Albedo] and transmitted once through Earth atmosphere.

Arcichovsky V.M. (1912) suggested to look for chlorophyll absorption in the Earthshine spectrum, with the aim to calibrate chlorophyll in the spectrum of other planets, but at these times, Earthshine observations did not have sufficient spectral resolution (Tikhoff 1914; Danjon 1928). Earthshine shows Rayleigh scattering in the Earth atmosphere and allowes to predict that from space Earth is seen as blue. The red side of the Earth reflectance spectrum shows the presence of $\mathrm{O}_{2}$ and $\mathrm{H}_{2} \mathrm{O}$ absorption bands, while the blue side clearly shows the Huggins and Chappuis ozone $\left(\mathrm{O}_{3}\right)$ absorption bands.

\section{Results obtained}

The first detections of vegetation from the Earthshine spectrum were obtained by Arnold et al. (2002) at Haute-Provence, and by Woolf et al. (2002) at the Tucson. Observations made at ESO NTT (Hamdani et al. 2006) obtain a VRE lower than previous studies, which were near 8-10\% when Africa and Europe light the Moon (Arnold et al. 2002). The present results are from 3 to $4 \%$ when Africa faces the Moon and $1.3 \%$ when the Pacific faces the Moon. Even with these lower values, VRE differs over Pacific Ocean vs. Africa, thus allowing detection of vegetation on Earth. These observations also show significant variations in Rayleigh scattering depending on cloud cover, implying that Earth as 'pale blue dot' can be almost white.

\section{Importance of observations from Dome C, Antarctica}

Observations of Earthshine can be done during the first and last days of the lunar cycle. From intermediate Earth's latitudes, observations of the waxing Moon are possible in the evening, and of the waning Moon in the morning, in both cases twilight observations. Only at high latitudes it is possible to observe the Moon in the first or the last days of the cycle during several hours, sometimes even all the day long. This happens at Dome $\mathrm{C}$ $\left(75^{\circ} 06^{\prime} \mathrm{S}, 123^{\circ} 21^{\prime} \mathrm{E}\right)$, about six times per year. During one observing run, continents and oceans successively face the Moon and the variations of the VRE corresponding to successive 'landscapes' of the planet Earth can be detected. A small telescope and low resolution spectrograph can be used to detect VRE in Earthshine spectra. Observations are carried out by one of us (E.A.) since March 2006.

\section{References}

Arcichovsky, V. M. 1912, Don Cesarevitch Alexis a Novotcherkassk, Vol. 1, no. 17, 195

Arnold, L., Gillet, S., Lardière, O., Riaud, P., \& Schneider, J. 2002, A\&GA, 352, 231

Danjon, A. 1928, Ann. Obs. Strasbourg, 2, 165

Hamdani, S., Arnold, L., Foellmi, C., Berthier, J., Billeres, M., Briot, D., François, P., Riaud, P., \& Schneider, J. 2006, A\&A, 460, 617

Tikhoff, G.A. 1914, Mitteillungen der Nikolai-Haupstrenwarte zu Pulkovo, no. 62, Band VI2, 15 Sagan, C., Thompson, W. R., Carlson, R., Gurnett, D., \& Hord, C. 1993, Nature, 365, 715

Woolf, N. J., Smith, P. S., Traub, W. A., \& Jacks, K. W. 2002, ApJ, 574, 430 